Article

\title{
Electrostatic Discharge Current Linear Approach and Circuit Design Method
}

\author{
Pavlos K. Katsivelis *, Georgios P. Fotis, Ioannis F. Gonos, Tryfon G. Koussiouris and \\ Ioannis A. Stathopulos
}

School of Electrical and Computer Engineering, National Technical University of Athens, 9 Iroon Polytechniou Str., Zographou 157 80, Athens, Greece; E-Mails: gfotis@gmail.com (G.P.F.); igonos@ieee.org (I.F.G.); tkous@softlab.ntua.gr (T.G.K.); stathop@power.ece.ntua.gr (I.A.S.)

* Author to whom correspondence should be addressed; E-Mail: pkatsivelis@gmail.com; Tel.: +30-210-772-3472; Fax: +30-210-772-3504.

Received: 14 October 2010; in revised form: 3 November 2010 / Accepted: 3 November 2010 / Published: 8 November 2010

\begin{abstract}
The Electrostatic Discharge phenomenon is a great threat to all electronic devices and ICs. An electric charge passing rapidly from a charged body to another can seriously harm the last one. However, there is a lack in a linear mathematical approach which will make it possible to design a circuit capable of producing such a sophisticated current waveform. The commonly accepted Electrostatic Discharge current waveform is the one set by the IEC 61000-4-2. However, the over-simplified circuit included in the same standard is incapable of producing such a waveform. Treating the Electrostatic Discharge current waveform of the IEC 61000-4-2 as reference, an approximation method, based on Prony's method, is developed and applied in order to obtain a linear system's response. Considering a known input, a method to design a circuit, able to generate this ESD current waveform in presented. The circuit synthesis assumes ideal active elements. A simulation is carried out using the PSpice software.
\end{abstract}

Keywords: electrostatic discharge current; linear system; approximation method; electrostatic discharge circuit 


\section{Introduction}

Electrostatic Discharge (ESD) is not only a natural phenomenon of great interest, but also a great danger for contemporary electronic devices. Both the great energy stored in the charged body and the rapid rise and fall time parameters of the ESD current can pose a great threat for the body-victim [1-5]. A typical equation describing the ESD current is set by the IEC 61000-4-2 [6]. However, this equation, whose graph can be seen in Figure 1, is incompatible with the simplified circuit of the ESD generator also included in [6]. A PSpice software simulation can prove this [7].

Figure 1. Typical waveform of the output current of an ESD generator [6].

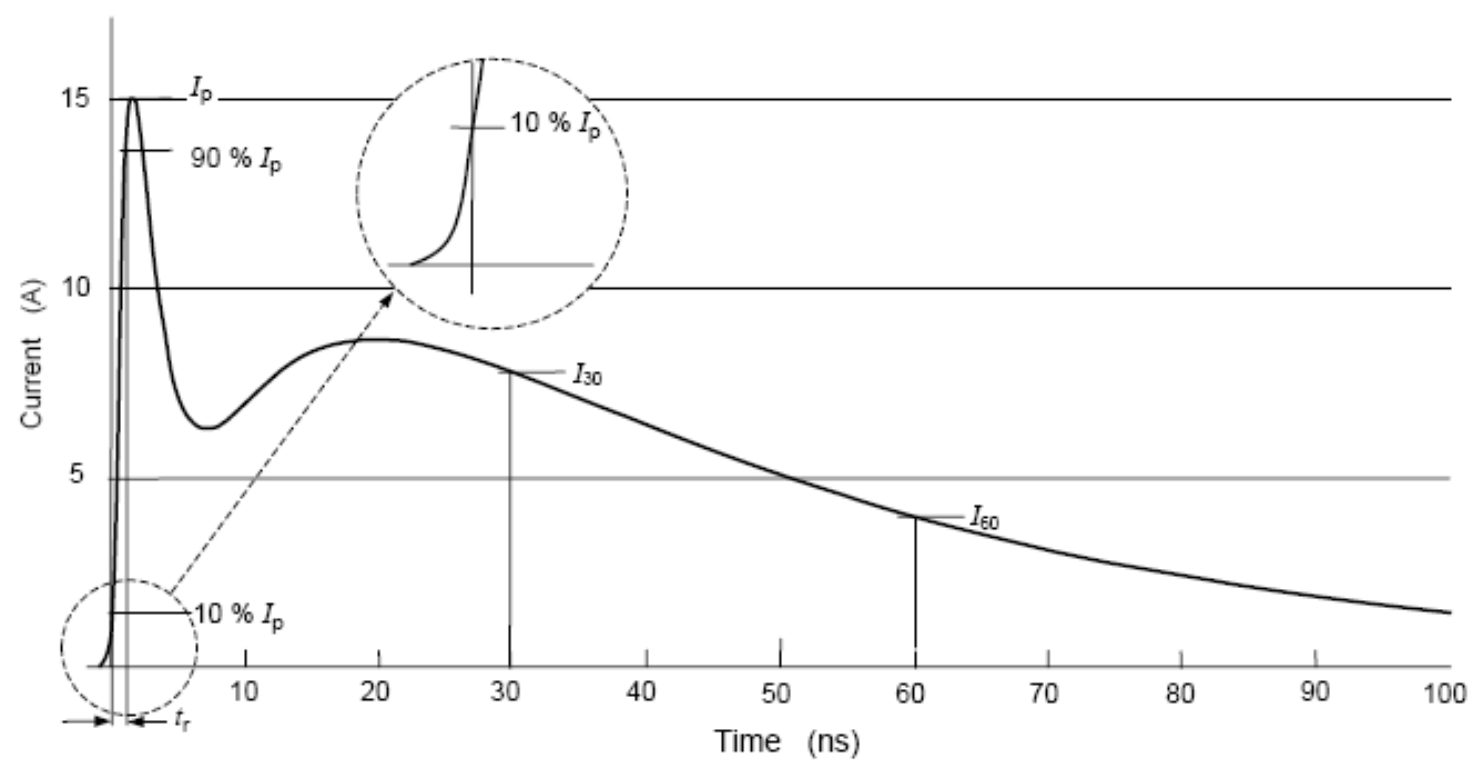

There have been various publications which propose improved circuits for ESD generators [8-11]. A modified ESD generator with a reference waveform very close to the one defined by the IEC Standard [6], as well as an equation for the reference waveform, have been proposed in [8]. In another work [9], the adopted human body model (HBM) is divided into 11 elemental blocks and is treated by the diakoptic method in analogy with network theory, using PSpice software. A recent publication [10] clarifies the parameters that govern the discharge current waveforms of ESD generators, proposing an equivalent circuit model based on the structure and dimensions of the ESD generator. In [11] two accurate and efficient models for electrostatic discharge generators are proposed, which permit reproduction of the discharge current in the contact mode taking into account the load effect.

In this work, the equation included in the last version of the IEC 61000-4-2 [6], is considered. Treating Prony's Method as reference a quite advanced approximation method is developed. By the use of this method, a linear equation of the ESD current is obtained and an active circuit that realizes this optimal approximation is proposed.

The paper is structured as follows. In Section 2 the equation of the discharge current is introduced. In Section 3 the approximation (modified) method is described and applied to determine the transfer function of the system that produces the ESD waveform as a step response. In Section 4 an active circuit is proposed and its output is determined and compared to the one described in the Standard. Section 5 contains the conclusions. 


\section{The Discharge Current of ESD}

\subsection{The Need for an Analytical and Accurate Equation for the ESD Current}

The ESD current must follow the shape of an HBM pulse as shown in Figure 1. This pulse is divided into two parts: The first peak, known as the "Initial Peak", is caused by the discharge of the arm, and generates the maximum current. The second peak is caused by the discharge of the body. The rise time of the initial peak is between $0.6 \mathrm{~ns}$ and $1 \mathrm{~ns}$, and its amplitude depends on the charging voltage of the ESD simulator. Figure 2 shows a simplified circuit of an ESD generator [6].

Figure 2. Simplified circuit of the ESD generator [6].

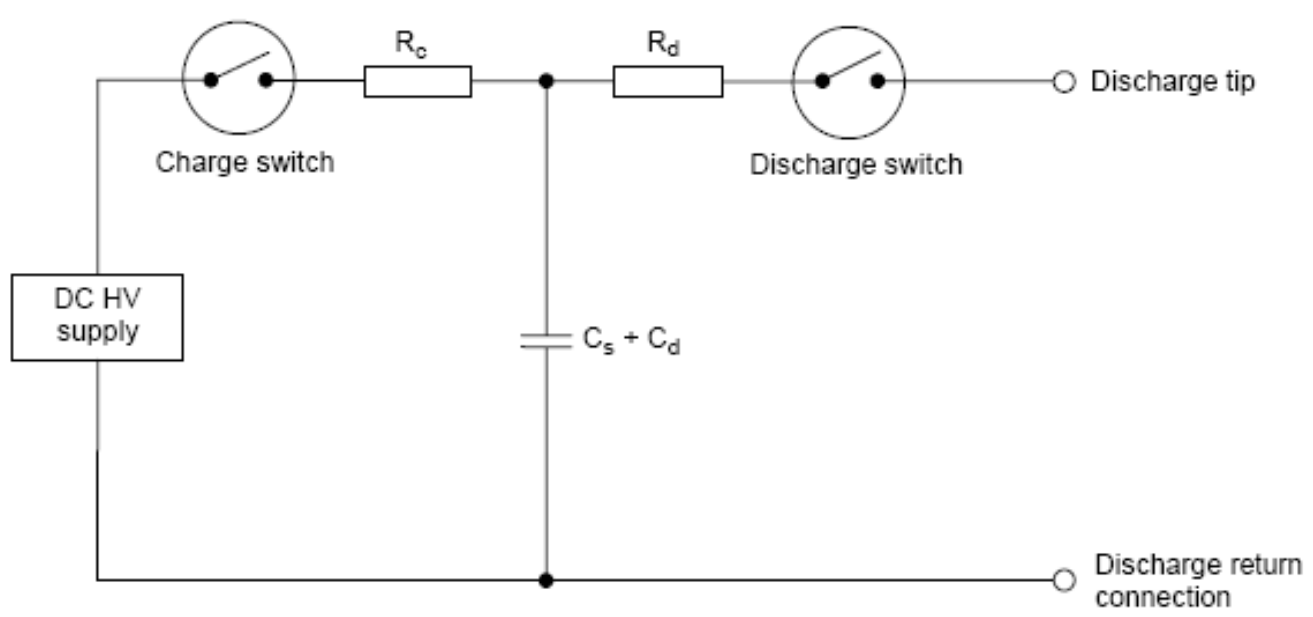

According to the IEC Standard the circuit consists of the charging resistor $R_{\mathrm{c}}(50-100 \mathrm{MOhms})$, the energy-storage capacitor $C_{\mathrm{S}}$, the distributed capacitance $C_{\mathrm{d}}\left(C_{\mathrm{S}}+C_{\mathrm{d}}=150 \mathrm{pF} \pm 10 \%\right)$, and the discharge resistor $R_{\mathrm{d}}$, representing the resistance of the skin (330 Ohms $\pm 10 \%$ ). The equipment under test (EUT) is to be connected to the open tips (Discharge tip, Discharge return connection). It should be mentioned that the reference model of the ESD waveform is the human-metal discharge. It is clear that $R_{\mathrm{d}}$ is the total skin resistance and not only that of the skin very close to the discharge point. The value of the energy-storage capacitor $C_{\mathrm{S}}$ is representative of the electrostatic capacitance of the human body. Also, in Figure 2 two switches are depicted. When the first switch is closed, the second is opened in order for the capacitor $\left(C_{\mathrm{S}}+C_{\mathrm{d}}\right)$ to be charged. After the capacitor is charged the first switch opens and the second closes, so the electrostatic discharge on the EUT occurs.

Figure 3 is obtained by simulations using PSpice software for an ohmic load of 2 Ohms, for a DC charging voltage of $+4 \mathrm{kV}$. One can easily observe that Figure 3 is different from the waveform defined by the IEC Standard [6], shown in Figure 1. The maximum ESD current value is 12 A, whereas the IEC Standard [6] defines $15 \mathrm{~A}$.

Furthermore, in this simulation, there is only one peak at the beginning (hand discharge), in contrast to the typical ESD current (hand and body discharge). This results in errors in obtaining voltages and currents in the EUT when simulations or experiments are carried out. In order to minimize these errors, a new circuit is needed for the ESD generator. 
Figure 3. Current waveform of the circuit of Figure 2 using PSpice.

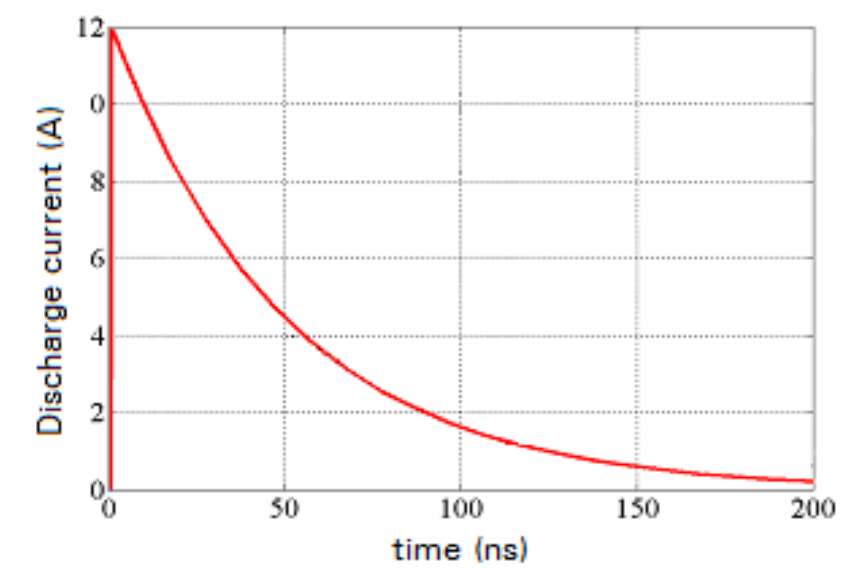

\subsection{Equation of the ESD Current}

In the last version of the IEC 61000-4-2, an analytical formula for the ESD current is included. It has been proven that this is the most accurate equation, which describes the ESD current $[12,13]$. This waveform is given by the following formula:

$$
i(t)=i_{1}(t)+i_{2}(t)
$$

where

$$
\begin{aligned}
& i_{1}(t)=\frac{I_{1}}{\left[-\frac{\tau_{1}}{\tau_{2}} \cdot\left(\frac{n \tau_{2}}{\tau_{1}}\right)^{1 / n}\right]} \cdot \frac{\left(\frac{t}{\tau_{1}}\right)^{n}}{1+\left(\frac{t}{\tau_{1}}\right)^{n}} \cdot e^{-\left(\frac{t}{\tau_{2}}\right)} \\
& i_{2}(t)=\frac{I_{2}}{e^{\left[-\frac{\tau_{3}}{\tau_{4}} \cdot\left(\frac{n \tau_{4}}{\tau_{3}}\right)^{1 / n}\right]} \cdot \frac{\left(\frac{t}{\tau_{3}}\right)^{n}}{1+\left(\frac{t}{\tau_{3}}\right)^{n}} \cdot e^{-\left(\frac{t}{\tau_{4}}\right)}}
\end{aligned}
$$

When examining the ESD current of a discharge under a charging voltage of $+4 \mathrm{kV}$, the values of the parameters are: $I_{1}=16.6 \mathrm{~A}, I_{2}=9.3 \mathrm{~A}, \tau_{1}=1.1 \mathrm{~ns}, \tau_{2}=2 \mathrm{~ns}, \tau_{3}=12 \mathrm{~ns}, \tau_{4}=37 \mathrm{~ns}$, and $n=1.8$ [6]. The IEC Standard [6] defines intervals for the values of these waveform parameters of the discharge current that an ESD generator has to follow. These parameters are [6]:

- the first peak current $\left(I_{\mathrm{p}}\right)$;

- the rise time $\left(t_{\mathrm{r}}\right)$, that is the time duration between the moment when the value of the ESD current is for the first time equal to $10 \%$ of its maximum value and the moment when the ESD current reaches for the first time $90 \%$ of its maximum value;

- the current at $30 \mathrm{~ns}\left(I_{30 \mathrm{~ns}}\right)$, that is the value of the current $30 \mathrm{~ns}$ after the moment when the current has reached for the first time $10 \%$ of its maximum value, and

- the current at $60 \mathrm{~ns}\left(I_{60} \mathrm{~ns}\right)$, that is the value of the current $60 \mathrm{~ns}$ after the moment when the current has reached for the first time $10 \%$ of its maximum value. 
The intervals for the values of the parameters, defined by the IEC Standard [6], are presented in Table 1. An evaluation of the IEC 61000-4-2 equation for the ESD current, with respect to the values of crucial parameters of the ESD current, is shown in Table 1.

Table 1. Parameter values of IEC 61000-4-2 equation for the ESD current. Discharge voltage $+4 \mathrm{kV}$.

\begin{tabular}{lcc}
\hline Parameter & $\begin{array}{l}\text { Values of the Parameters of } \\
\text { the ESD Current Defined by } \\
\text { the IEC 61000-4-2 Standard }\end{array}$ & $\begin{array}{l}\text { Values of the Parameters of the ESD } \\
\text { Current Calculated Using the IEC 61000-4-2 } \\
\text { Equation for the ESD Current }\end{array}$ \\
\hline$I_{\mathrm{p}}(\mathrm{A})$ & $15 \pm 15 \%$ & 15.14 \\
$t_{\mathrm{r}}(\mathrm{ns})$ & $0.8 \pm 25 \%$ & 0.88 \\
$I_{30 \mathrm{~ns}}(\mathrm{~A})$ & $8 \pm 30 \%$ & 7.83 \\
$I_{60 \mathrm{~ns}}(\mathrm{~A})$ & $4 \pm 30 \%$ & 3.98 \\
\hline
\end{tabular}

\section{The Approximation Methods}

The Prony Method approximates a function by a sum of exponentials. It has been used in many applications such as analysis of power converter signals, monitoring of electrical machines, analysis of fault currents and signal predicting algorithms [14-17]. The Prony method is an approximation method which intends to approach a data set by an equation which will present values of a given sub-group of the data set.

As in our case, the Prony method has to be applied on a sample of the given curve which we wish to approximate. A sufficient sampling mode is of essence. This is vital to ensure that the necessary data of the curve will be available, in order not to lose any useful information of its behavior, and avoid distortions. After proper sampling has been applied these points are treated as input in the Prony algorithm (or the modified algorithm which will be shown hereunder). The outcome is a linear systems approximation given in the form of an exponential series.

\subsection{The Basic Idea}

Briefly, the method works as follows: let $g_{d}(t)$ be a continuous function. Let us consider the values of $g_{d}(t)$ as a set of equally spaced points $t=k T, k=0,1, \ldots$ and form the matrix:

$$
P(N, M)=\left[\begin{array}{cccccc}
g_{d}(0) & g_{d}(T) & . & . & . & g_{d}[(M-1) T] \\
g_{d}(T) & g_{d}(2 T) & \cdot & . & . & g_{d}(M T) \\
\cdot & \cdot & & & & \cdot \\
\cdot & \cdot & & & \cdot \\
\cdot & \cdot & & & \cdot \\
g_{d}[(N-1) T] & g_{d}(N T) & . & . & . & g_{d}[(N+M-2) T]
\end{array}\right]
$$

If $g_{d}(t)$ is the impulse response of a linear, time invariant system of finite order $n$, it can be written as in (5):

$$
g_{d}(k T)=\sum_{i=1}^{n} A_{i} \exp \left(s_{i} k T\right)=\sum_{i=1}^{n} A_{i} z_{i}^{k}
$$


where

$$
z_{i}=\exp \left(s_{i} T\right)
$$

Let us define the polynomial in $z$ having roots $z_{i}, i=1,2, \ldots, n$, as in (7):

$$
\psi(z)=\prod_{i=1}^{n}\left(z-z_{i}\right)=z^{n}+\sum_{m=0}^{n-1} b_{m} z^{m}
$$

Then for $N, M$ greater than $n$ :

$$
\operatorname{rank} P(N, M)=n
$$

This is equivalent to saying that any $n+1$ columns of the matrix $P(N, M)$ are linearly dependent and for its first $n+1$ columns the following relation holds:

$$
\left[\begin{array}{cccccc}
g_{d}(0) & g_{d}(T) & \cdot & \cdot & \cdot & g_{d}[(M-1) T] \\
g_{d}(T) & g_{d}(2 T) & \cdot & \cdot & \cdot & g_{d}(M T) \\
\cdot & \cdot & & & \cdot \\
\cdot & \cdot & & \cdot \\
\cdot & \cdot & & & \cdot \\
g_{d}[(N-1) T] & g_{d}(N T) & \cdot & \cdot & \cdot & g_{d}[(N+M-2) T]
\end{array}\right]\left[\begin{array}{c}
b_{0} \\
b_{1} \\
\cdot \\
\cdot \\
\cdot \\
b_{n-1}
\end{array}\right]=-\left[\begin{array}{c}
g_{d}(n T) \\
g_{d}(n T+T) \\
\cdot \\
\cdot \\
\cdot \\
g_{d}[(N+n-1) T]
\end{array}\right]
$$

where $b_{i}$ are the coefficients of $\psi(z)$, as in (6). Furthermore, the system of equations:

$$
\left[\begin{array}{cccccc}
1 & 1 & \cdot & \cdot & \cdot & 1 \\
z_{1} & z_{2} & \cdot & \cdot & \cdot & z_{n} \\
\cdot & \cdot & & & \cdot \\
\cdot & \cdot & & & \cdot \\
\cdot & \cdot & & & \cdot \\
z_{1}^{N-1} & z_{2}^{N-1} & \cdot & \cdot & \cdot & z_{n}^{N-1}
\end{array}\right]\left[\begin{array}{c}
A_{0} \\
A_{1} \\
\cdot \\
A_{n}
\end{array}\right]=-\left[\begin{array}{c}
g_{d}(0) \\
g_{d}(T) \\
\cdot \\
\cdot \\
\cdot \\
g_{d}(N T-T)
\end{array}\right]
$$

is consistent and has a solution.

\subsection{The Prony Method}

In the general case $g_{d}(t)$ can be approximated by the impulse response of a linear, time invariant system of finite order, say $g(t)$ [18]. The function $g(t)$ is a interpolation of order $n$, when equation (11) holds:

$$
g_{d}(k T)=g(k T) \quad k=0,1,2, \ldots, 2 n-1
$$

To determine the order $n$ of the approximation, the rank of the matrix $P(N, M)$ has to be determined [18]. This can be obtained by examining the determinant of the submatrix $P(k, k)$ consisting of the first $k$ rows and the first $k$ columns of $P(N, M)$ for $k=1,2, \ldots$, where $n$ is the dimension of the last non zero minor. However since $g_{d}(t)$ does not correspond to a linear time invariant system, the order $n$ is considered as the minimum $n$ so that:

$$
|\operatorname{det} P(n+1, n+1)|<e_{q}
$$


This is equivalent to saying that $e_{q}$ is treated as an equivalent zero for the approximation and $n$ columns of the submatrix $P(n, n)$ are linearly independent. To determine $z_{i}$ 's, the coefficients of $\psi(z)$ are determined from (9) if $N$ is replaced by $n$. Having obtained the roots of $\psi(z), A_{i}$ 's are determined from (10) if $N$ is replaced by $n$.

\subsection{The Modified Method}

A more accurate method of tracking the transfer function will be demonstrated in the following. The aim is to use the maximum possible number and not only the first $2 n$ samples in order to obtain the approximation. The matrix $P(N, M)$ is again formed and linearly independent columns in the submatrix $P(N, k)$ consisting of the first $N$ rows and the first $k$ columns of $P(N, M)$ are sought.

If the first $n+1$ columns are linearly dependent, the minimum singular value of the matrix $P(N, n+1)$ is equal to zero. Similar to the standard method, since $g_{d}(t)$ does not correspond to a linear time invariant system, the order $n$ of the approximation is defined as the minimum $n$ such that:

$$
\left|\sigma_{\min }(N, n+1)\right|<e_{q}
$$

Again $e_{q}$ is treated as an equivalent zero for the approximation. Equations (9) and (10) now do not possess solutions but approximations of the solutions, obtained by minimizing the Euclidian norm of the errors using generalized inverses. So the vectors containing the parameters $A_{i}$ and $S_{i}$ are determined.

\subsection{Applying the Approximation Methods}

There is an inherent difficulty in applying any approximation method to approximate $i(t)$. This waveform has a very fast mode and a slow mode so if a small sampling period $T$ is chosen, the slow mode is not taken into account while if a large period $T$ is selected the fast mode is ignored. To overcome this difficulty the Prony Method is applied separately on each summand of (1) and the overall approximation is the sum of the two approximations. Since for the Laplace transform the following property holds:

$$
L\{f(a t)\}=F\left(\frac{s}{a}\right)
$$

to avoid writing very large numbers time scaling by the factor $10^{9}$ is used, so the time corresponds to seconds. For the approximation of $i_{1}(t)$, the sampling period $T$ and the error $e_{q}$ have been taken equal to 0.16 and $10^{-6}$ respectively, for the Prony Method and, 0.084 and $10^{-7}$ for the modified approximation method. Note that, for the Prony method, the sampling period $T$ has to be taken such that will produce a system of desired order. So, it is a matter of scanning the examined interval in such detail that will produce a manageable order of the system. On the other hand, the modified method does not follow the same procedure with Prony method. The selected points do not represent values of the outcome curve. Instead, best fitting among them is achieved by generalizes inverses. Thus the choice of $T$ is more matter of how accurate the description will be, assuming a given system order. So, $T$ is taken so small until the improvement in no longer significant. The resulting transfer functions of the systems whose impulse responses approximate $i_{1}(t)$ are: 
- Prony Method:

$$
g_{1 P}=\frac{27.5944}{s+0.3749}-\frac{-13.7972+14.8589 i}{s+1.7783-1.2697 i}+\frac{-13.7972-14.8589 i}{s+1.7783+1.2697 i}
$$

- Modified Method:

$$
g_{1 M P}=\frac{48.3895}{s+0.4879}-\frac{63.2844}{s+1.3352}+\frac{7.3867-2.2784 i}{s+(3.403+17.892 i)}+\frac{7.3888+2.2784 i}{s+(3.403-1.789 i)}+\frac{0.0972}{s+20.06}
$$

For the approximation of $i_{2}(t)$ the sampling period $T$ and the error $e_{q}$ have been taken equal to 14.75 and $10^{-6}$ respectively, for the Prony Method and, 10 and $10^{-7}$ for the modified approximation method. The resulting transfer functions of the systems whose impulse responses approximate $i_{2}(t)$ are:

- Prony Method:

$$
g_{2 P}=\frac{27.2173}{s+0.0314}+\frac{27.2174}{s+0.0762}
$$

- Modified Method:

$$
g_{2 M P}(s)=\frac{19.9542}{s+0.2607}-\frac{12.1536}{s+8.3700}-\frac{22.8566}{s+17.8801}+\frac{15.4674}{s+31.1830}
$$

Then the transfer functions of the linear time invariant systems that approximate the ESD current as their impulse responses are as follows:

- Prony Method:

$$
g_{P}(s)=2.2126 \frac{(s+46.88)}{(s+0.0314)(s+0.3749)(s+0.0762)} \frac{\left(s^{2}+0.1742 s+0.0233\right)}{\left(s^{2}+3.5570 s+4.7740 i\right)}
$$

- Modified Method:

$$
\begin{aligned}
g_{M P}(s)= & \frac{-0.0001}{s+0.0262} \cdot \frac{s+0.4241}{s+0.4117} \cdot \frac{s+0.0875}{s+0.0724} \cdot \frac{s-572.1058}{s+20.0599} \cdot \frac{s+2028.7500}{s+1.3352} \\
& \cdot \frac{s+14.1242}{s+0.4879} \cdot \frac{s+6.3195}{s+0.1480} \cdot \frac{s^{2}+0.1516 s+0.0337}{s^{2}+6.8054 s+14.7796}
\end{aligned}
$$

The outcome of the standard Prony Method is plotted together with (1) in Figure 4.

It can be observed that there is a fairly large deviation between the IEC 61000-4-2 equation and the impulse response that the Prony Method produced. The values of the relative error norms are presented in Table 2. In the time interval between $2 \mathrm{~ns}$ and $60 \mathrm{~ns}$, which is considered to be the one of the highest interest, the Prony Method gives an average relative error norm which reaches the value $5.69 \%$ ( $0.5 \mathrm{nsec}$ step was used for the relative error calculation).

In Figure 4 the impulse response produced by the modified method is also plotted. Compared to the plot of (1), one can observe an excellent result. In Table 2 the relative errors of the method are also shown. The improvement of the relative error is considerable. 
Figure 4. Plot of the IEC 61000-4-2 equation for the ESD current at $4 \mathrm{kV}$, together with the outcome of the standard and modified Prony Method.

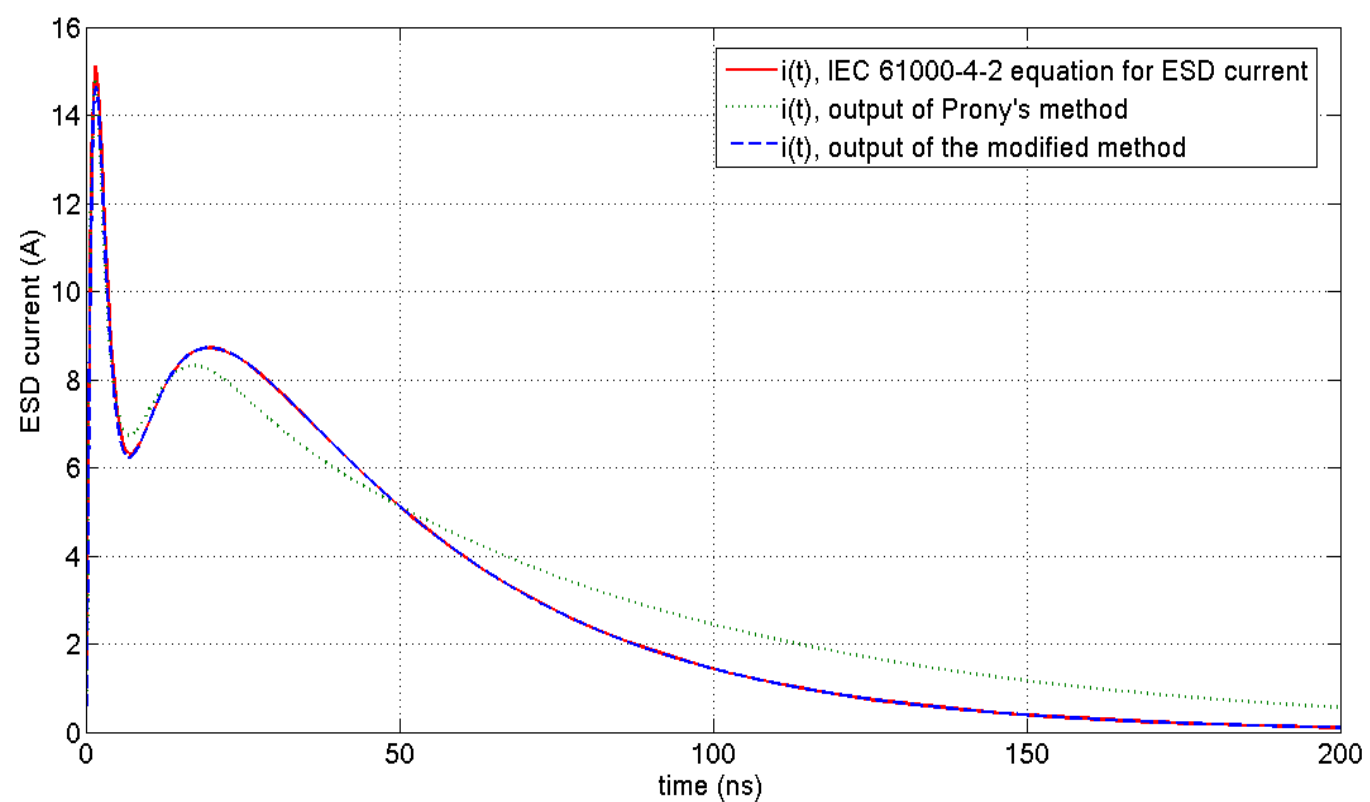

Table 2. Relative error between the impulse response given by each method and the IEC 61000-4-2 equation for the ESD current.

\begin{tabular}{lccc}
\hline \multirow{2}{*}{ Method } & \multirow{2}{*}{ Time Interval } & \multicolumn{2}{c}{ Relative Error Norm } \\
\cline { 3 - 4 } & & Maximum & Average \\
\hline \multirow{2}{*}{ Prony Method } & $2-60 \mathrm{~ns}$ & $50.43 \%$ & $5.69 \%$ \\
& $0-200 \mathrm{~ns}$ & $50.43 \%$ & $21.23 \%$ \\
\hline \multirow{2}{*}{ Modified Method } & $2-60 \mathrm{~ns}$ & $12.19 \%$ & $0.45 \%$ \\
& $0-200 \mathrm{~ns}$ & $12.19 \%$ & $0.32 \%$ \\
\hline
\end{tabular}

\subsection{The Transfer Function}

The Prony Method as well as the Modified Method approximate the ESD current by the impulse response of a system $S$ having transfer function $g(s)$. If the step response is required for the approximation, the transfer function $G(s)$ of the resulting system $S$ ' has to satisfy the relation:

$$
Y(s)=g(s) L\{\delta(t)\}=g(s)=G(s) L\{u(t)\}=\frac{G(s)}{s}
$$

Taking into account (14), if (20) is applied to (21), the required transfer function is:

$$
\begin{gathered}
G(s)=-0.0001 \cdot \frac{\frac{s}{10^{9}}}{\frac{s}{10^{9}}+0.0262} \cdot \frac{\frac{s}{10^{9}}+0.4241}{\frac{s}{10^{9}}+0.4117} \cdot \frac{\frac{s}{10^{9}}+0.0875}{\frac{s}{10^{9}}+0.0724} \cdot \frac{\frac{s}{10^{9}}-572.1058}{\frac{s}{10^{9}}+20.0599} \\
\frac{\frac{s}{10^{9}}+2028.7500}{\frac{s}{10^{9}}+1.3352} \cdot \frac{\frac{s}{10^{9}}+14.1242}{\frac{s}{10^{9}}+0.4879} \cdot \frac{\frac{s}{10^{9}}+6.3195}{\frac{s}{10^{9}}+0.1480} \cdot \frac{\left(\frac{s}{10^{9}}\right)^{2}+0.1516 \frac{s}{10^{9}}+0.0337}{\left(\frac{s}{10^{9}}\right)^{2}+6.8054 \frac{s}{10^{9}}+14.7796}
\end{gathered}
$$




\section{The Circuit}

The above transfer function is realized as a cascade connection of biquads. The pole-zero pairing and the place of each biquad were selected using standard rules. For each one of the first seven factors, a circuit of the following form was considered:

Figure 5. Form of the inverting connection used to design circuit that corresponds to factors 1 to 7 of the transfer function.

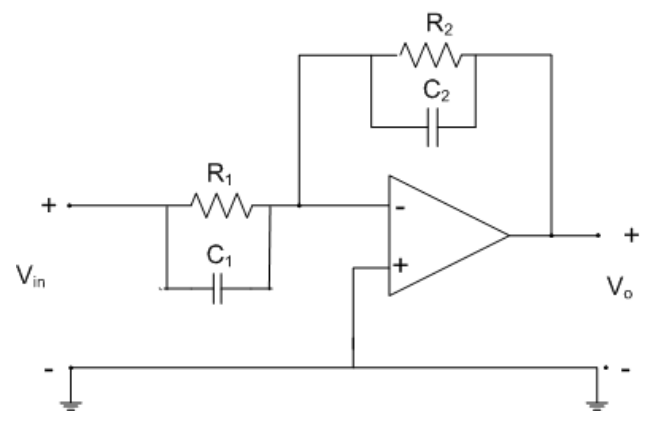

A Friend's circuit [18], depicted in Figure 6, was used for creating a circuit that corresponds to the last factor of the expression of the transfer function $G(s)$.

Figure 6. Form of the connection used to design circuit that corresponds to the last factor of the transfer function: Friend's circuit.

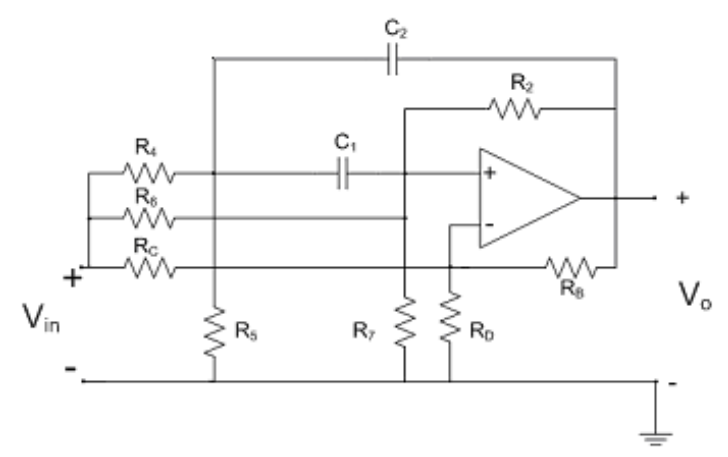

The chain-connected biquads are shown in Figure 7. The values of the components of the circuit are shown in Tables 3 and 4.

Figure 7. The circuit that produces the ESD current at $+4 \mathrm{kV}$.

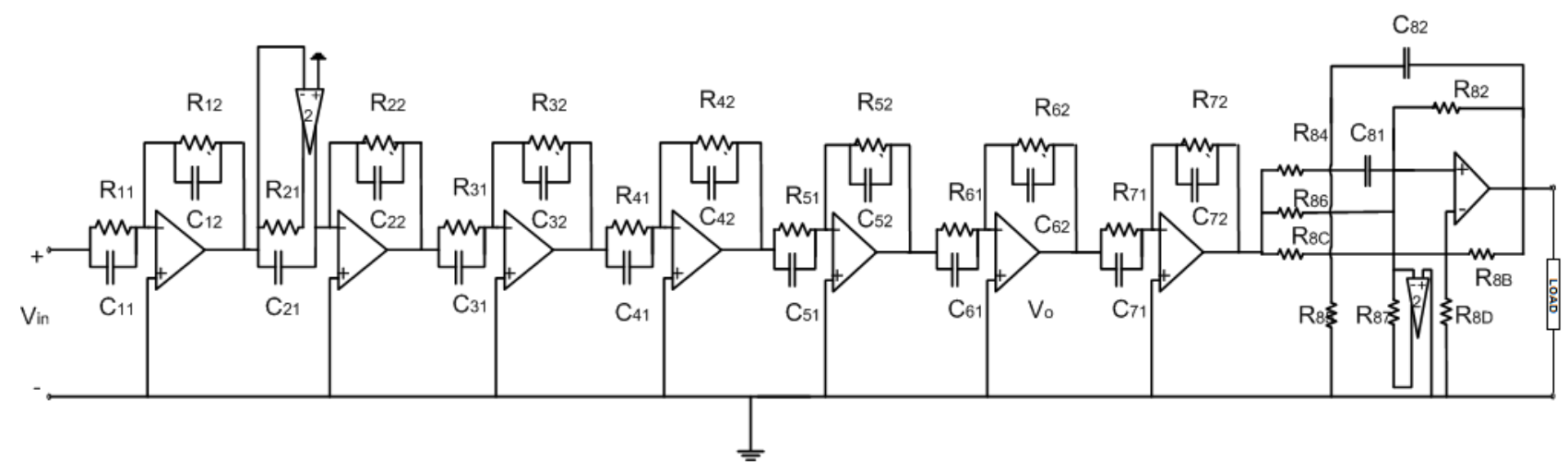


Table 3. Component values (corresponding to factors 1-7).

\begin{tabular}{cccc}
\hline $\mathbf{I}$ & $\boldsymbol{R}_{\mathbf{i}, \mathbf{1}}(\boldsymbol{\Omega})$ & $\boldsymbol{R}_{\mathbf{i}, \mathbf{2}}(\boldsymbol{\Omega})$ & $\boldsymbol{C}_{\mathbf{i}, \mathbf{1}}=\boldsymbol{C}_{\mathbf{i}, \boldsymbol{(} \mathbf{p F})}$ \\
\hline 1 & $\infty$ & 3.84 & $10^{4}$ \\
2 & 1.77 & 49.85 & 1 \\
3 & 8.85 & 11.95 & $10^{3}$ \\
4 & 2.86 & 5.59 & $10^{3}$ \\
5 & 1.56 & 32.07 & $10^{2}$ \\
6 & 0.73 & 20.49 & $10^{2}$ \\
7 & 0.38 & 74.91 & 10 \\
\hline
\end{tabular}

Table 4. Component values (corresponding to factor 8 -Friend's circuit).

\begin{tabular}{ccccc}
\hline$R_{8,2}(\Omega)$ & $R_{8,4}(\Omega)$ & $R_{8,5}(\Omega)$ & $R_{8,6}(\Omega)$ & $C_{8,1}(\mathrm{nF})$ \\
\hline 0.32 & 1.15 & 1.00 & 1.01 & 1.00 \\
\hline$R_{8,7}(\Omega)$ & $R_{8, \mathrm{~B}}(\Omega)$ & $R_{8, \mathrm{C}}(\Omega)$ & $R_{8, \mathrm{D}}(\Omega)$ & $C_{8,2}(\mathrm{nF})$ \\
\hline 0.46 & 1.00 & 1.00 & 0.00 & 1.00 \\
\hline
\end{tabular}

The output of the simulation is presented in Figure 8, along with the plot of (1) and the upper and lower limits set for the ESD current waveform by the Standard [6] in Annex A. The average relative error that appears between the output of the simulated circuit and the (1) equation is $4.11 \%(0.5 \mathrm{nsec}$ step for error calculation). Therefore, the circuit designed is considered to operate very well. The circuit's output meets all requirements for the ESD current waveform included in the Standard [6].

Figure 8. Plot of the output of the circuit of Figure 9, together with the IEC 61000-4-2 Standard equation for the ESD current at $+4 \mathrm{kV}$ and the tolerance set by the Standard.

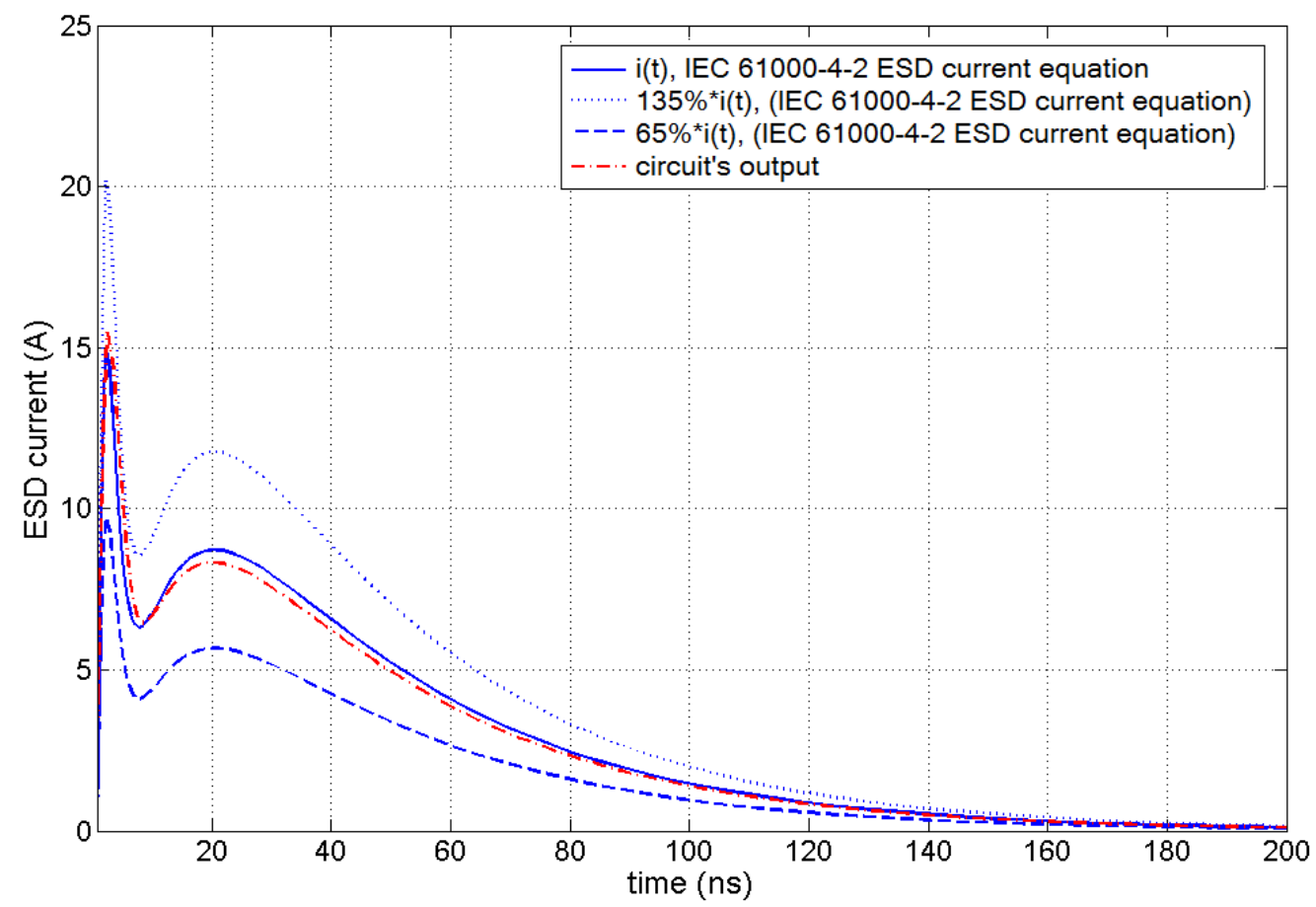




\section{Conclusions}

The problem of the insufficiency of the circuit proposed by IEC 61000-4-2 Standard [6] for the ESD generator to produce such an ESD current waveform as the Standard [6] itself demands is addressed in this paper. A new approximation method was presented and employed for the approximation of the IEC 61000-4-2 ESD current as the step response of a linear time invariant system. The method had to be applied separately on each one of the summands due to the stiffness of the waveform, and gave very accurate results for the ESD current waveshape, delivering a system of 9th order. An ideal element design of a correspondent active circuit took place in the form of chain connected biquads. Simulations were successful; the ESD current output is very close to the theoretical one.

\section{References}

1. Barth, J. Measurements of real ESD threats that have been ignored too long. IEEE Instrum. Meas. Mag. 2005, 8, 61-63.

2. Fujiwara, O.; Taka, Y. Gap Breakdown field caused by air discharge through hand-held metal piece from charged human-body. In Proceedings of the 2006 4th Asia-Pacific Conference on Environmental Electromagnetics, Dalian, China, 1-4 August 2006; pp. 103-106.

3. Lou, L.; Liou, J.J. An Improved Compact Model of Silicon-Controlled Rectifier (SCR) for Electrostatic Discharge (ESD) Applications. IEEE Trans. Electron Devices 2008, 55, 3517-3524.

4. Huang, J.; Liu, S.; Wang, X.; Zhou, F.; Wang, L.; Gao, Y. Intrinsic Characterization of Human Metal ESD: Current, Electromagnetic Field and Displacement Current. In Proceedings of the 2006 4th Asia-Pacific Conference on Environmental Electromagnetics, Dalian, China, 1-4 August, 2006; pp. 518-520.

5. Yuan, Z.; Li, T.; He, J.; Chen, S.; Zeng, R. New Mathematical Descriptions of ESD Current Waveform Based on the Polynomial of Pulse Function. IEEE Trans. Electromagn. Compat. 2006, 48, 589-591.

6. International Electrotechnical Commission. International Standard IEC 61000-4-2: "Electromagnetic Compatibility (EMC), Part 4: Testing and Measurement Techniques, Section 2: Electrostatic Discharge Immunity Test-Basic EMC Publication"; 2008.

7. Fotis, G.P.; Gonos, I.F.; Iracleous, D.P.; Stathopulos, I.A. Mathematical analysis and simulation for the electrostatic discharge (ESD) according to the EN 61000-4-2. In Proceedings of the 39th International Universities Power Engineering Conference, Bristol, UK, 6-8 September 2004; pp. 228-232.

8. Wang, K.; Pommerenke, D.; Chundru, R.; Doren, T.V.; Drewniak, J.L.; Shashindranath. A. Numerical modeling of electrostatic discharge generators. IEEE Trans. Electromagn. Compat. 2003, 45, 258-270.

9. Amoruso, V.; Helali, M.; Lattarulo, F. An improved model of man for ESD applications. J. Electrostat. 2000, 49, 225-244.

10. Fujiwara, O.; Tanaka, H.; Yamanaka, Y. Equivalent circuit modeling of discharge current injected in contact with an ESD-gun. Electr. Eng. Jpn. 2004, 149, 8-14. 
11. Caniggia, S.; Maradei, F. Circuital and numerical modeling of electrostatic discharge generators. In Proceedings of the 14th IAS Annual Meeting, IEEE Industry Applications Conference, Kowloon, Hong Kong, 8 August 2005; pp. 1119-1123.

12. Fotis, G.P.; Gonos, I.F.; Stathopulos, I.A. Determination of the discharge current equation parameters of ESD using genetic algorithms. IEE-Electron. Lett. 2006, 42, 797-799.

13. Fotis, G.P.; Gonos, I.F.; Assimakopoulou, F.; Stathopulos, I.A. Applying genetic algorithms for the determination of the parameters of the electrostatic discharge current equation. Inst. Phys. Publishing Meas. Sci. Technol. 2006, 17, 2819-2827.

14. Carriere, R.; Moses, R.L. High Resolution Radar Target Modeling Using a Modified Prony Estimator. IEEE Trans. Antennas Propog. 1992, 40, 13-18.

15. Pierre, D.A.; Trudnowski, D.J.; Hauer, J.F. Identifying linear reduced-order models for systems with arbitraryinitial conditions using Prony signal analysis. IEEE Trans. Autom. Control 1992, 37, 831-835.

16. Qi, L.; Qian, L.; Cartes, D.; Woodruff, S. Initial results in Prony analysis for harmonic selective active filters. In Proceedings of IEEE Power Engineering Society General Meeting, Montreal, Canada, 18-22 June 2006; p. 6.

17. Li, D.; Cao, Y.; Wang, G. Online identification of low-frequency oscillation in power system based on fuzzy filter and Prony algorithm. In Proceedings of International Conference on Power System Technology, Chongqing, China, 22-26 October 2006; pp. 1-5.

18. Daryanani, G. Principles of Active Network Synthesis and Design; Wiley: New York, NY, USA, 1976.

(C) 2010 by the authors; licensee MDPI, Basel, Switzerland. This article is an open access article distributed under the terms and conditions of the Creative Commons Attribution license (http://creativecommons.org/licenses/by/3.0/). 